\title{
Calibration of safety factors for offshore wind turbine support structures using fully coupled simulations
}

\author{
A. Morató ${ }^{a}$, and S. Sriramula ${ }^{a^{*}}$ \\ ${ }^{a}$ School of Engineering, University of Aberdeen, Aberdeen, United Kingdom \\ *Corresponding author: \\ Dr Srinivas Sriramula, Senior Lecturer \\ School of Engineering, University of Aberdeen \\ Aberdeen, AB24 3UE, UK. \\ Phone: +44 (0)1224 272000 \\ Email: s.sriramula@abdn.ac.uk
}




\title{
Calibration of safety factors for offshore wind turbine support structures using fully coupled simulations
}

\author{
The offshore wind industry experienced a boost during the last decade in terms \\ of size of wind farms and rated capacity of the wind turbines: towers are getting \\ taller and blades are getting longer, constantly facing new and complex \\ challenges. Because of the relative immaturity of the wind industry, and the fact \\ that the offshore design standards stemmed from the oil and gas industry, it is \\ generally acknowledged that the reliability levels achieved, although not very \\ well understood, might result in partial safety factors not optimal for OWT. This \\ paper addresses this situation by studying the reliability levels delivered by the \\ current standards and assessing the validity of the safety factors through a \\ reliability-based code calibration. The combination of the low probability of \\ failure imposed on the design of OWTs and the computational cost of the aero- \\ elastic time-domain simulations brings out the need to develop new approaches \\ for reliability analyses. In this paper, the reliability analysis is performed using a \\ Kriging surrogate model to approximate the load-effect from the aero-elastic \\ simulations converting expensive-to-evaluate limit state functions to explicit \\ functions. Subsequently, a calibration of the safety factors is carried out using \\ the probabilistic models from literature. The approach is applied to an industry- \\ reference turbine and support structure. The results showed very low \\ probabilities of failure for the most severe design cases and confirm that the \\ safety factors from the IEC are mostly adequate.
}

Keywords: Offshore wind turbines structures; code calibration; reliability; Kriging; aeroelastic simulations

\section{Introduction}

Offshore wind is considered one of the most attractive renewable energy resources [1]: unlike onshore wind, it takes advantage of the availability of large areas with high and consistent wind speeds, lower turbulence and less wind shear [2]. Oceans are thus becoming the main target to utilise this new and immense potential.

Even though the offshore wind industry is developing rapidly, it is also relatively immature as its origin only dates back to the 1990s. In fact, it was not until the last decade that the increasing demand led to a boost in terms of the size of the farms and the rated capacity of the turbines [3]. In the last few years, Offshore Wind Turbines (OWTs) have been developed even into the 7 to $12 \mathrm{MW}$ range.

It is however not only the size of turbines, but the unique environments of the new sites - waves, wind and seabed composition - that are defining the challenges in the design of support structures, including towers and foundation. The increment in turbine capacity directly requires a higher resistance and thus larger tower diameters and 
deeper foundations, making the soil-structure interaction become increasingly challenging.

As a direct consequence of this rapid development and due to the growing demand in renewable energy production, the immaturity of the industry becomes clear with regards to its own codes and standards. These were originally taken from the Oil and Gas industry, maintaining the corresponding reliability levels, safety factors and design methodologies. They were arguably not developed to deliver the optimum structure (which in case of an unmanned unit should aim at minimising total expenditure) but to ensure safety above all since most Oil and Gas offshore structures are inhabited most of the time. Here lies a first and major obstacle in the optimisation of the support structures for wind turbines.

The leading technical standards for the design of wind turbines are developed by the International Electrotechnical Commission (IEC). In particular, structural design is done according to IEC 61400-1 [4] and IEC 61400-3 [5]. It is believed though, that the defined partial load factors in these standards are not necessarily consistent with the resistance partial factors defined in the codes for structural element design [6]. Additionally, there is no standard method to allow for site-specific load factors, which can be appropriate since environmental hazard curves may vary between sites. Other standards used for the OWT design are developed by Det Norske Veritas (DNV) [7] and the American Petroleum Institute (API) [8]. However, these standards are borrowed from the oil industry, drilling stations or onshore wind turbine design codes. It is thus believed that the way in which these OWTs are designed may lead to conservative systems which are therefore needlessly expensive. Besides, because the support structures play an important role in the final cost of the system, the safety factors have a direct effect on the Levelised Cost of Energy (LCOE) and this is key for increasing the competitiveness of the offshore wind versus other energy sources.

With that in mind, the high reliability levels achieved in previous work [9] lead to an unavoidable reconsideration of the role and consistency of the safety factors in the codes for the design of OWT. In fact, studying the reliability levels implied by the current LRFD-based standards is necessary considering that the offshore wind industry is relatively new, and it is still evolving. Note that the first edition of IEC 61400-3 was issued in 2009. Besides, to the authors' best knowledge, there is no study in the public domain that analyses the reliability levels delivered by such codes and therefore the efficiency of the structural designs. Thus, in this paper the reliability levels achieved by such standards are explored along with the validity of such factors and appropriate reliability targets for these structures.

Most of the public work regarding safety factors focuses on blades, thus in this paper the validity of the safety factors is studied by exploring the reliability levels achieved by some of the severest Ultimate Limit State (ULS) cases from [10] focusing on the support structure from the Upwind project [11]. An approach based on aero-hydro-servo-elastic 
simulations and the surrogate Kriging model is implemented for reliability assessment, see [9]. The current reliability levels delivered by the IEC 61400-3 are studied through a reliability-based code calibration approach considering model and physical uncertainties. The study focuses on ULS using a limit state equation based on the local buckling failure.

\section{Current reliability levels}

RECOFF [12] was the first project that addressed the complexity of the combination of the Oil and Gas offshore standards and the existing onshore wind energy standards. A series of recommendations were published and developed later on along with the IEC 61400-3. In this regard, the oil and gas industry was quite mature and standards for the design of bottom-supported offshore structures such as API RP 2A-LRFD [8] and API RP 2A-WSD [13] were well established, providing a good starting point of transition towards OWT standards. However, these standards were not intended for wind turbines, which are active machines rather than passive structures, and for support structures which are not only greatly influenced by aerodynamic loads, but also by the nonlinearities introduced by the controllers. Besides, from a structural design perspective, several factors have to be considered in the design of OWT support structures which are absent in their onshore counterparts, such as the interaction and uncertainties in the different sources of loading, the harsh saline environment, etc. In spite of the extended use of these standards, it is generally acknowledged that the reliability levels achieved are not very well understood and the resulting partial safety factors might not be optimal for OWT.

For manned and unmanned offshore steel jacket structures of oil and gas production, typically maximum annual probabilities of failure in the ranges of $10^{-5}$ to $5 \cdot 10^{-5}$ and $10^{-4}$ to $2 \cdot 10^{-4}$ respectively are generally accepted $[14,15]$; see also, e.g., DNV GL RP-C203 [16] and DNV GL RP-C210 [17]. Some studies [18-21] suggest that an implicit reliability index of 3.09 is used in the IEC 61400-1 for onshore wind, and [22] recommends 3.3, which corresponds to minor/moderate consequences of failure and moderate/high cost of safety measure for offshore structures that are unmanned or evacuated in severe storms and where other consequences of failure are not very significant.

In the last decade, some authors studied the possibility of a redefinition of the safety factors by estimating the reliability levels delivered with the current standards for the design of components of an OWT. For instance, Moriarty [23] studied the influence on the safety factors of the two methodologies used to extrapolate extreme loads and deflection given a small set of simulations. Also, authors such as Toft and Sorensen $[20,21,24]$ performed reliability-based calibration of safety factors for offshore wind turbines, mainly focusing on blade design. For example, the authors in [21] presented a probabilistic framework to incorporate results from small and full-scale tests on the reliability-based design approach. The tests were used to obtain stochastic models that 
subsequently led to optimal partial safety factor derivation. For the ultimate state, it was found that the number of tests greatly influences the reliability results, achieving a significant reduction of the safety factors when increasing the number of tests. In contrast, results based on the fatigue limit state showed that the current partial factors from the IEC [4] are adequate and that it is to be associated with the high uncertainty related to a simplified method such as Miner's rule. Similarly, the reliability of the blades is studied in [20], where a response surface along with nonlinear finite element analyses was used for reliability estimation of wind turbine blades. In this case, they focused on nonlinear failure modes due to buckling based on the Tsai-Wu failure criterion, making use of stochastic models for the material and load-effect as well. The results shown a lower probability of failure than the one implicitly used in IEC 61400-1, which, although the reliability level seemed highly dependent on the model uncertainties related to the failure criteria, shows that there is room for optimisation. With a different approach, Agarwal and Manual describe in $[25,26]$ a reliability-based investigation on the required safety factors to be used for fatigue design of steel structures in offshore wind turbines. The methodology formulates stochastic models for the strength and load parameters and includes the effect of some possible inspections during the design lifetime. Material SN-curves and Miner's rule were applied, and they conclude that significant reductions on the safety factors may be achieved with inspections during the design lifetime.

It is significant that, to the authors' knowledge, none of the previous literature has used fully integrated simulations to compute the load effects. In the end, the use of fully coupled simulations aims to obtain increased design certainty, or design maturity, through the consideration of the interaction between the loads applied to the global model's foundation structure and the wind turbine generator. Besides, research related to calibration of safety factors for ULS of the OWT support structures is non-existent or very scarce. With this in mind, the present paper proposes to include coupled simulations in a probabilistic approach with a view to explore the validity of the current safety factors.

\section{Formulation of the code calibration method}

Calibration of the safety factors of structural codes has been addressed in literature frequently, initially in [27-30]. Also, the Joint Committee on Structural Safety (JCSS) has been a prominent contributor in this direction: they provided general knowledge about the matter and published guides and notes for probabilistic risk assessment [31-33] and a probabilistic model code [34], which have been widely used in structural reliability and safety factor calibration [35].

A code calibration is performed when a superior method to assess the reliability is developed or there are significant improvements in the stochastic models involved in the design code. There are two main approaches for a code calibration: one maximises 
the expected utility function (by translating design variables into a measure of utility), while the other is set to achieve a constant target reliability (set prior to the calibration) for all the structures within the class to which the code is applicable.

The calibration of a code is generally done for a given class of structures, materials and/or loads. The pure code calibration is extensively explained in $[30,33,36,37]$. Depending on the approach taken for the code calibration, the process involves different stages. However, they all share the initial steps, as the following [14]:

\section{1) Structure class}

This first step requires a definition of the scope of the code. It is important to determine the range of the code accurately. An example of this step is to choose the class of structures that will be considered.

\section{2) Probabilistic code}

The second step is to formulate the stochastic models for the material parameters and actions as well as to obtain characteristic values, establish load configurations and cases, etc. An important work here is to quantify the uncertainty related to the mathematical models in terms of probability distributions, which will certainly affect the results of the calibration procedure.

\section{3) Goal of the code}

The objective of the code needs to be defined. Here, as mentioned before, the procedure can be either a maximisation of the expected utility or the realization of certain reliability index. This is directly related to levels 2 and 3 reliability methods, as explained in [36].

\section{4) Importance weighting}

In this step, each design event gets an associated weight for the evaluation function depending on the frequency of occurrence of such events. This process can be different for various design states; that is, in fatigue limit state, these weights are related to the global probability of occurrence of any representative event, whereas for ultimate limit states, the weights can be selected in such a way that a given combination of load case and failure mode is considered more likely, given prior experiences or engineering judgement.

\section{5) Measure of fit}

This step is only included in the approach that aims at achieving a constant target reliability level. The idea is to use a function that quantifies how far a code format is from its target reliability at each iteration of the optimisation process. Such a function is known as a penalty function. There are a couple of penalty functions used commonly in the literature: a basic function, $\mathrm{M}$, that equally penalises values regardless of whether they are above or below the target: 
$M\left(\beta, \beta_{T}\right)=\left(\beta-\beta_{T}\right)^{2}$

where $\beta$ is the actual code reliability level and $\beta_{T}$ is the target of reliability; or an exponential penalty function that confers larger penalties to under-designs than to over-designs.

$M\left(\beta, \beta_{T}\right)=c\left(\beta-\beta_{T}\right)+\exp \left[-c\left(\beta-\beta_{T}\right)\right]-1$

where $c$ is a constant that can be taken as 4.35 [36]. However, it has been proven that the final result is not very sensitive to the choice of the penalty function [37].

6) Optimisation of the code format

Within the approach to obtain a constant reliability level, this step aims at minimising the penalty function from step 5 . With the cost-benefit formulation, the optimisation is carried out with a function that includes benefits and costs as an explicit function of the safety factors, see the following subsection 4.1.

\section{Optimisation of the code}

The following formulations are explained in this section:

- Direct calibration of partial safety factors through a cost-benefit formulation

- Optimisation of safety factors based on a target reliability

\subsection{Cost-benefit formulation}

This approach is based on a direct calibration of the partial safety factors from a costbenefit point of view. In this case the code is calibrated such that the benefits are maximised formulating the calibration as a decision-making problem. This method requires identification of benefits and costs as a function of certain design variables, where the costs are subdivided into construction, maintenance and failure costs. A general formulation is shown below, further detail may be found in [14].

$\max _{\gamma} W(z(\gamma))=\sum_{j=1}^{L} \omega_{j}\left[B_{j}-C_{I j}(z(\gamma))-C_{R j}(z(\gamma))-C_{F j} P_{F j}(z(\gamma))\right]$

s.t. $\gamma_{i}^{l} \leq \gamma_{i} \leq \gamma_{i}^{u}, i=1, \ldots, m$

where $z$ defines the design variable as a function of $\gamma ; \gamma=\left(\gamma_{1}, \ldots, \gamma_{m}\right)$ are the $m$ partial safety factors to be calibrated and the $l$ and $u$ upper indices indicate the lower and upper bounds. The variable $L$ stands for the number of design cases/failure modes and $\omega$ is a weight indicating the relative importance of each of them. $B_{j}$ is the expected benefits either for the society or for the owner of the structure. $C_{I j}$ and $C_{R j}$ are the construction and repair/maintenance costs and $C_{F j}$ is the cost of failure, independent of $\gamma, P_{F j}$ is the probability of failure of the failure mode $j$ for a given safety factor $\gamma$. 
Each limit state function for failure modes is written as follows:

$g_{j}\left(X, p_{j}, z\right)=0, j=1, \ldots, L$

where $X$ represents the stochastic variables with their probabilistic models, $p_{j}$ is a vector with deterministic parameters and $z$ is the design variable.

The optimal design variable $z$ is obtained by minimising the construction costs, using the characteristic values $x_{c}$ of the stochastic variables $X$ :

$\min _{Z} C_{I j}(z)$

s.t. $G_{j}\left(x_{c}, p_{j}, z, \gamma\right) \geq 0, j=1, \ldots, L$

$z^{l} \leq z \leq z^{u}$

where $G_{j}$ represents the deterministic design equation related to the limit state function $g_{j}$ and act as constraints. The repair costs can easily be included in the optimisation of Equation (5).

In other words, a process for such calibration would follow as: first, for a given set of $\gamma$ and the characteristic values of the stochastic variables $x_{c}$, a first optimisation is performed using the design variable $z$, to minimise construction costs in Equation (5). Then, with the optimal value for $z$, the probability of failure is obtained with the set of limit state functions and with all stochastic models in Equation (4). Finally, with the already obtained $z(\gamma)$ and the probability of failure $P_{F j}(z(\gamma))$, a second optimisation is performed in Equation (3) aiming at maximising the difference between benefits and costs. As noted, this process includes two optimisation processes in Equations (3) and (5) and if the probability of failure is computed using FORM, then this will include an extra optimisation. Thus, if the number of design variables, failure modes and partial safety factors increase, the computation of the optimisation problem can become highly complex.

\subsection{Constant reliability level formulation}

The idea of this approach is that when the structures within the class that the code is calibrated for are designed using the new calibrated partial safety factors, the reliability level achieved will be as close as possible to the target of reliability.

As mentioned before, the optimal safety factors are obtained by numerical solution of the optimisation problem in Equation 1. First, with a given safety factor $\gamma_{i}$ a design-tolimit is performed with the deterministic design equation using characteristic values. Then the $j$ reliability index of the corresponding limit state function is computed using FORM/SORM removing the safety factors and using the probabilistic models of the stochastic variables. Finally, the $\beta_{j}$ obtained is compared to the reliability target, if the result is not optimal the algorithm proceeds with the following iteration. 
Both methodologies are summarised in flow charts in Figure 4-1. Because of the lack of precise models for the relationships between costs and safety factors, the latter approach is used in this study in the application example.

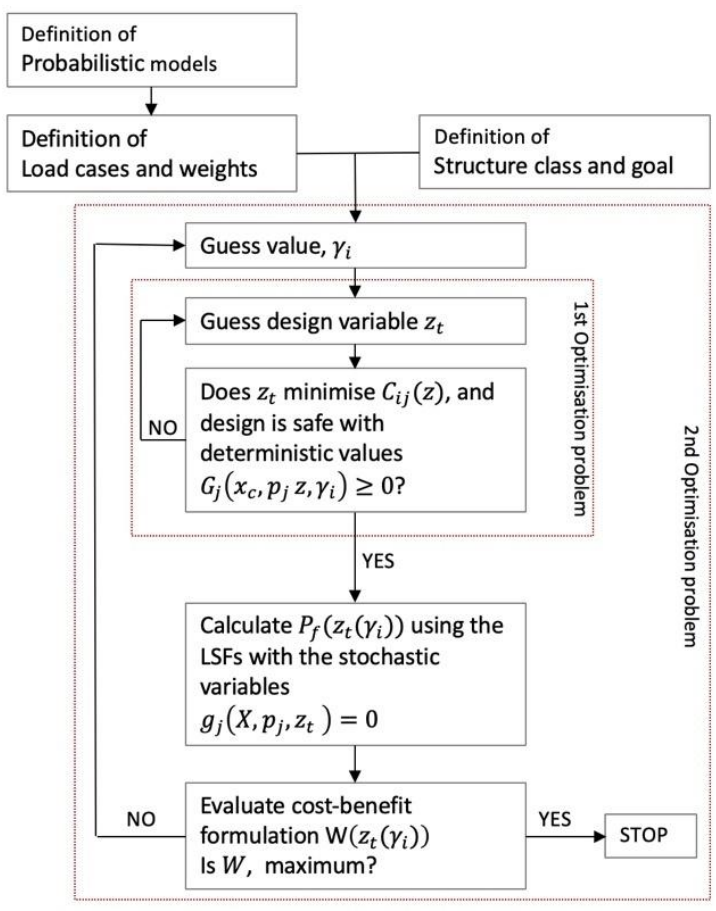

a)

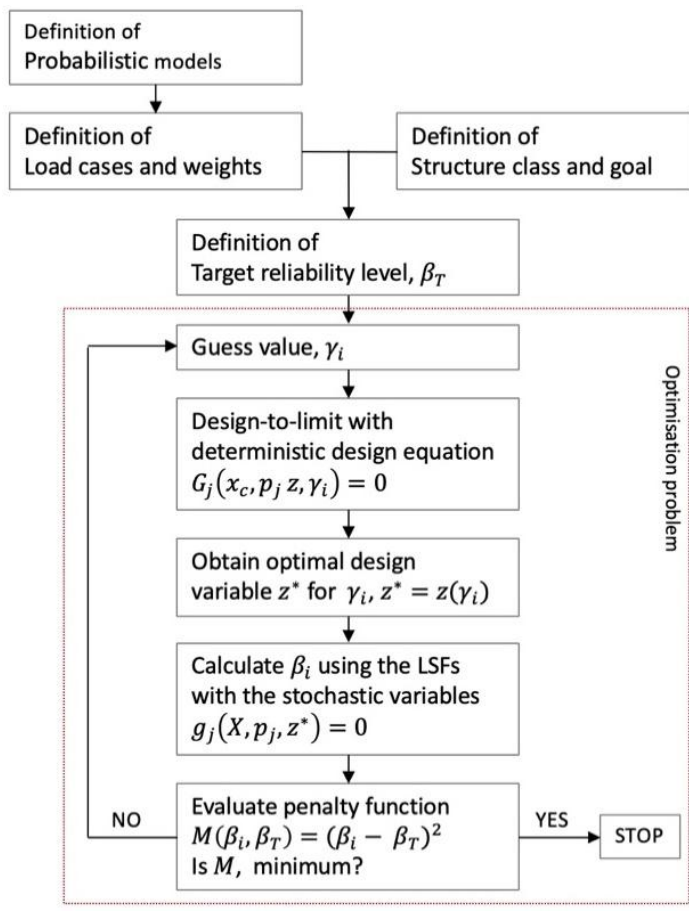

b)

Figure 4-1. Flow charts for both approaches. a) Cost-benefit formulation. b) Constant target reliability

\section{Target reliabilities}

It is difficult to judge whether the reliability index of a structure obtained using a set of stochastic variables with their probabilistic models is sufficient unless there is a reference for comparison. Such reference may be established by the definition of an optimal or "best practice structure". The idea of a best practice is that if the structure is designed to that reliability level, then the structure is optimal according to the agreed conventions.

There are different ways to determine the best practice: one could be to use an existing standard as starting point or alternatively it can be directly derived by experts. Furthermore, if a more rational approach is preferred, then the reference may be established based on an economic decision theory by considering costs and benefits. In this case the optimal solution could be determined from the following formulation; for further details refer to [38].

$$
E[B]=I\left(1-P_{f}\left(C_{D}\right)-C_{D}-C_{F} P_{f}\left(C_{D}\right)=I-C_{D}-\left(I+C_{F}\right) P_{f}\left(C_{D}\right)\right.
$$

where $I$ is the income, $P_{f}$ the probability of failure and $C_{D}$ and $C_{F}$ are costs of risk treatment and cost of failure relatively. Note that risk treatment is defined here as any 
risk-reducing measure. Note, as well, that $P_{f}$ is a function of the cost reduction measure. The optimal criterion for values of risk reduction costs is stated as:

$\frac{\partial E[B]}{\partial C_{D}}=-1-\left(I+C_{F}\right) \frac{\partial P_{f}\left(C_{D}\right)}{\partial C_{D}}$

It is possible to include the consequences of loss of human lives in the previous formulation through a Life Quality Index, see [38, 39].

JCSS [41] also provides recommendations for target reliability indexes for ULS and SLS, see Table 5-1 and Table 5-2. These are based on a one-year-reference period and associated to the stochastic models recommended by them [41].

Table 5-1. Recommended target reliability indices related to 1-year reference period and ULS

\begin{tabular}{|l|l|l|l|}
\hline $\begin{array}{l}\text { Relative cost of safety } \\
\text { measure }\end{array}$ & High & Normal & Low \\
\hline $\begin{array}{l}\text { Minor consequences of } \\
\text { failure }\end{array}$ & $\beta=3.1\left(P_{f} \approx 10^{-3}\right)$ & $\beta=3.7\left(P_{f} \approx 10^{-4}\right)$ & $\beta=4.2\left(P_{f} \approx 10^{-5}\right)$ \\
\hline $\begin{array}{l}\text { Moderate } \\
\text { consequences of failure }\end{array}$ & $\beta=3.3\left(P_{f} \approx 5 \cdot 10^{-4}\right)$ & $\beta=4.2\left(P_{f} \approx 10^{-5}\right)$ & $\beta=4.4\left(P_{f} \approx 10^{-5}\right)$ \\
\hline $\begin{array}{l}\text { Large consequences of } \\
\text { failure }\end{array}$ & $\beta=3.7\left(P_{f} \approx 10^{-4}\right)$ & $\beta=4.4\left(P_{f} \approx 5 \cdot 10^{-5}\right)$ & $\beta=4.7\left(P_{f} \approx 10^{-6}\right)$ \\
\hline
\end{tabular}

Table 5-2. Recommended target reliability indices related to 1-year reference period and SLS

\begin{tabular}{|l|l|l|l|}
\hline $\begin{array}{l}\text { Relative cost of safety } \\
\text { measure }\end{array}$ & High & Normal & Low \\
\hline $\begin{array}{l}\text { Target index } \\
\text { (irreversible SLS) }\end{array}$ & $\beta=1.3\left(P_{f} \approx 10^{-1}\right)$ & $\beta=1.7\left(P_{f} \approx 5 \cdot 10^{-2}\right)$ & $\beta=2.3\left(P_{f} \approx 10^{-2}\right)$ \\
\hline
\end{tabular}

Although these reliability targets are useful as a general guideline for common structures, OWTs are very distinctive to traditional structures. Offshore structures for wind turbines have many particularities, i.e. the number and type of load cases is currently being discussed and developed, there are many stochastic variables whose probabilistic models are still being quantified that may have significant impact on reliability calculations.

Thus, in the following section, a design-to-limit offshore wind turbine structure is studied to obtain the reliability levels achieved by following the aforementioned offshore standard. Next, the second approach for the code calibration is applied to explore validity of the current safety factors.

\section{Application example}

\subsection{Benchmark and site-specifics}

In this section, the monopile from the UpWind [42] project is chosen to confirm the results seen in [9]. The Upwind monopile is preferred over the OC3 one because the latter was created for code comparisons; it was not so much 'designed', but rather 
'specified' based on industry trends and information from other reference turbines. On the other hand, the Upwind project reviewed the design criteria of such structures and carried out a DLCs-based design, for both ultimate and fatigue cases from the standards $[4,5]$. The design involved fully integrated time domain analysis for a large number of DLCs, including extreme and fatigue cases. Among the requirements for the design of the structure was to maintain enough clearance between blades and the support structure to avoid collision, and to keep the tower top motions within predefined limits to avoid damage in components of the wind turbine. To satisfy these requirements, several criteria were formulated regarding natural frequencies, strength and deformations.

The monopile from the upwind project is extensively described in [42]. The final design consists of a foundation pile with a bottom diameter of $6 \mathrm{~m}$ and a conical section tapering to a top diameter of $5.5 \mathrm{~m}$. The embedded length is $24 \mathrm{~m}$ and the total length is $54 \mathrm{~m}$. The transition piece has an outer diameter of $5.8 \mathrm{~m}$ and a total length of 18.7 $\mathrm{m}$. The tower has a length of $68 \mathrm{~m}$, leading to a hub height of $85.2 \mathrm{~m}$. All parts of the structure are constructed with segments of constant thickness, varying diameter and different lengths, see Figure 6-1.

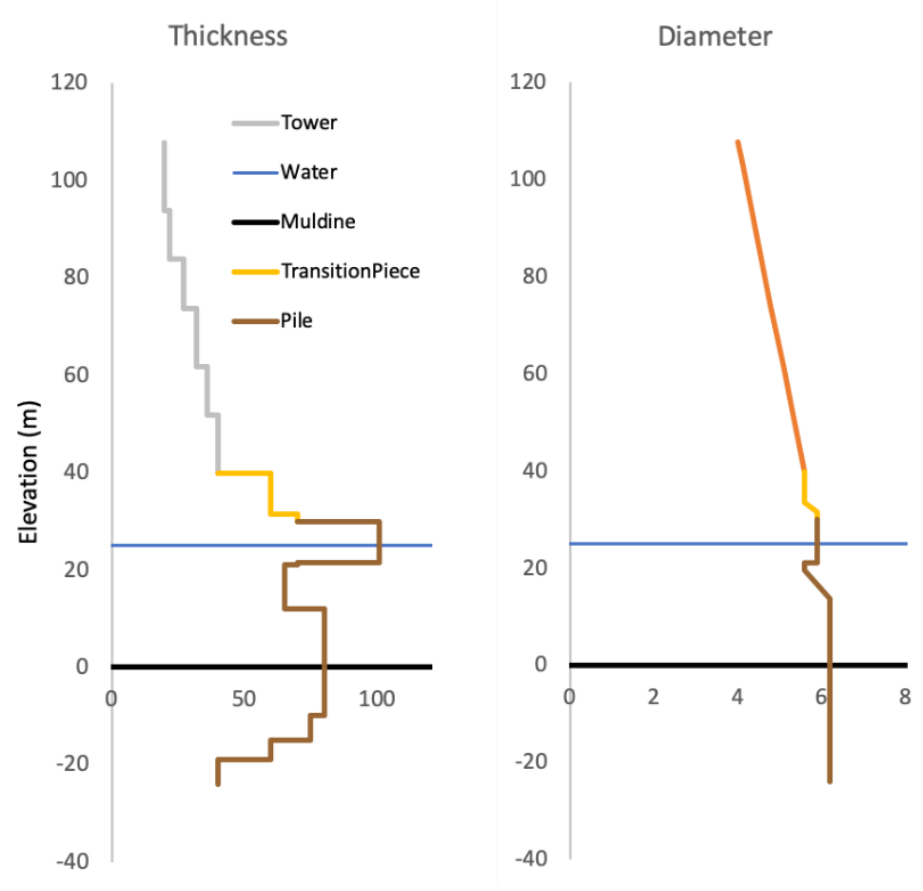

Figure 6-1. Properties of the Upwind structure from [42]

The metocean data used in this study is the same as that was used in the design basis for the design of the Upwind monopile: it corresponds to the K13 Shallow Water Site, for which all processed data are available in [11]. The site is in the Dutch North Sea and has a water depth of $25 \mathrm{~m}$. The location is associated with a Class $1 \mathrm{C}$ from the IEC 61400-1 [4]. 


\subsection{Failure modes}

According to the design process followed when the structure was designed, some of the most common failure modes are considered for an initial analysis. The idea is to perform a prior comparison to determine the most critical failure mode, which will be used in the subsequent sections for reliability analysis and code calibration. The considered failure modes are related to structural performance, which are the Von Mises stress reaching yield, plastic yielding and local buckling. The blade crashing against the tower is another ULS failure considered, although it is not related to the structural properties of the tower, since such an event would make the structure collapse. The design equations associated with the aforementioned failure modes are briefly summarised next.

The criterion of Von Mises' stress reaching yield at any point in the structure is a very conservative one but it has been widely used in the literature $[42,43]$. It states the constraint as:

$G=f_{y}-\sigma_{V M} \geq 0$

where $f_{y}$ is the yield strength and $\sigma_{V M}$ is the Von Mises' stress. The thickness effect has been considered for $f_{y}$.

With regards to plastic yielding, there are two design equations applicable: i) for axially loaded piles, the axial stress $\sigma_{A}$ is checked against the yield strength; and ii) for structures under bending, the bending moment $S_{M}$ at a given section must be smaller than a given parameter, which is calculated as the yield strength reduced by the section modulus $Z$, written as a function of the diameter $D$ and thickness $t$.

$$
\begin{aligned}
& G=f_{y}-\sigma_{A} \geq 0 \\
& G=Z f_{y}-S_{M}=\frac{1}{6}\left(D^{3}-(D-2 t)^{3}\right) f_{y}-S_{M} \geq 0
\end{aligned}
$$

Shell buckling is a very relevant failure mode for slender structures, but unlike the previous design equations, buckling lacks a uniformly prescribed formulation in all the standards and instead every code has its own approach for buckling assessment. Here different formulations for shell buckling are checked. The first design equation related to buckling, see $[24,44]$, is a simplified model for local buckling failure of an OWT support structure in shallow waters, which utilizes a reduction factor on the value of $Z f_{y}$ in a reformulation of the LSF in Equation (10).

$G=\frac{1}{6}\left(1-0.84 \frac{D}{t} \frac{f_{y}}{E}\right)\left(D^{3}-(D-2 t)^{3}\right) f_{y}-S_{M} \geq 0$ 
where $E$ is the Young's Modulus. This LSF is expected to be more demanding than the two previous ones as the resistant factor is reduced by a factor that depends on the ratio $D / t$.

Two other formulations are considered for local buckling, from the recommended practice DNV-RP-C202 [45] and AISC guidelines for steel structures [46]. The exact formulation of these is left to the reader in the original references.

The idea of this subsection is not to derive specific conclusions on the structural performance but to compare the severity of the deterministic design equations studied. For the sake of a consistent comparison, because some of the design equations use different moments and other stresses, the parameter of choice is the utilisation ratio. The utilisation ratio is defined as that between the resistance and the load or load effect and, in order to compute it, a set of realistic combination of forces and moments at the top of the tower must be taken.

The results are shown in Figure 6-2. As expected, the axial and bending plastic yielding give the lowest utilisation ratio of all design equations. It is interesting to see how three significantly different formulations for local buckling lead to such similar results. The ratio of Von Mises's stress reaching yield (Equation (9)) comes up close to that of shell buckling, and thus it should be considered as well, although it is considered a more conservative formulation than failure by shell buckling.

The failure modes for the following analyses are chosen to be the Von Mises's stress and the simplified expression for local buckling from Equation (11). The latter is preferred among all the buckling criteria as it leads to similar results with a simpler formulation. 


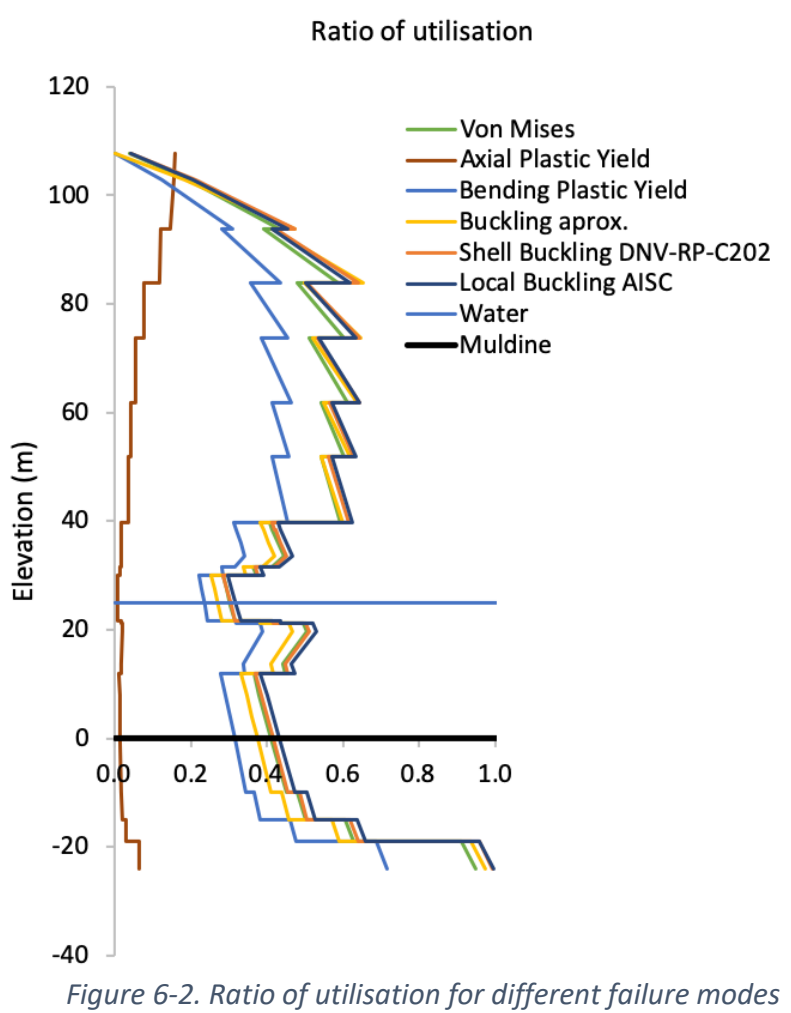

\subsection{Sensitivity analysis}

The Kriging model is a type of surrogate model that uses interpolation based on the assumption that there is a spatial correlation between the model predictions. The Kriging models do not assume an underlying global functional form as presumed in the polynomial regression models (e.g. first- or second-order polynomials) and can approximate arbitrary functions with high accuracy at global as well as local levels (Section 7.1 explains Kriging fundamentals further). Kriging is used herein to, given certain inputs, approximate the load effect from the interface between coupled simulations and finite element analyses. The outcome of the interface is then expressed as an explicit function of the input variables and used as a load effect in the limit state functions. This subsection aims at identifying those variables whose variability may have a significant effect on the outcomes of the simulations. The result from this sensitivity analysis is used a posteriori for quantification of the uncertainty related to the Kriging model.

The idea of this sensitivity analysis is to determine the stochastic variables with the greatest effect on different load effects considered in the LSFs chosen from the previous subsection. To do that, the metocean conditions from the power production load case 1.3 from the IEC 61400-3 [5] are taken as a basis, see [10] for further details on the DLC. Then, six variables that impact both resistance and loads are chosen: amongst the loads, the following are considered: wind speed at hub height $(\mathrm{Vhub})$, 
standard deviation of the Turbulence (stdT), significant wave height $(H s)$ and peak spectral period (Tp); whereas from the resistance variables, the thickness at tower base $(t t)$ and the Young's Modulus $(E)$ are studied. For each of these variables, a vector of 150 values is sampled within a range using Latin Hypercube Sampling Technique (LHST). For each element of the vector, an aero-hydro-servo-elasto simulation is run while the rest of the variables are taken as deterministic. This results in 150 output files for each stochastic variable producing an array of 150 elements for each of the outputs to analyse. Finally, the ratio between the Coefficient of Variation (CoV) of the input and the CoV is used to compare the effect on the output. This is done for the outputs bladetower clearance (TwrClr), tower top displacement (TopDx), overturning moment at tower base level (TwrMy) and mudline level (MudMy) and the Von Mises' stress at tower base level (TwrBsVM). Results of this analysis are presented in Figure 6-3.

The results show that, as expected, the wind speed at hub height has a significant impact on all the outputs considered. Variability in the standard deviation of the turbulence only has a substantial effect on TwrClr. It is also notable to see the influence of Young's Modulus and thickness on the tower top displacement and Von Mises' stress at tower base respectively. Nevertheless, the wind speed at hub height and thickness at tower base showed the largest influence on outputs that are implicit in the selected failure modes. While $E$ has a huge influence on the displacement of the tower top, this load effect is not considered in any of the chosen failure modes, and thus will be taken as deterministic. Since $H_{S}$ and $T_{p}$ are used to describe a wave spectrum, this will still add some implicit variability to the outputs but they will not be considered as stochastic variables in the model.

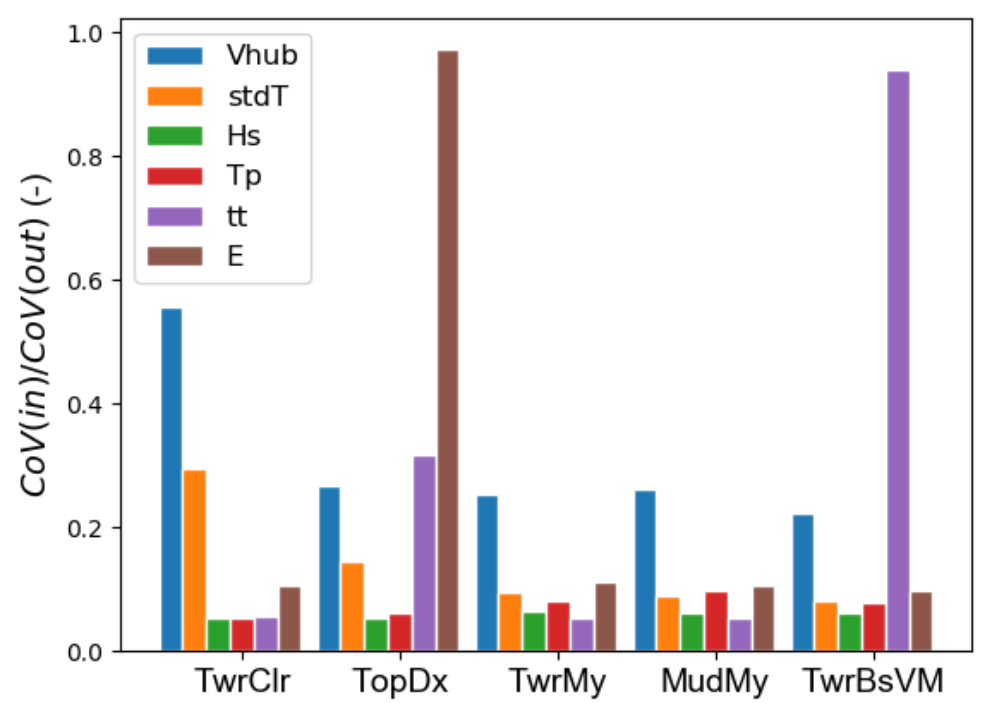

Figure 6-3. Ratio between the CoV of the input and CoV of the output for different stochastic variables and output channels. 


\section{Reliability analysis}

\subsection{Fundamentals of Kriging models}

If each evaluation of the LSF includes implicit aero-hydro-servo-elasto simulations, traditional reliability methods become highly inefficient. Thus, as in [9], the Kriging surrogate model is used in the reliability analyses to approximate the outputs of the aero-elastic simulations as an $\mathrm{n}$-dimensional surface. This approach transforms an implicit expensive-to-evaluate LSF to an explicit function with which Monte Carlo or FORM/SORM can be applied.

The idea of Kriging method is to estimate the value of the output from the sum of the weighted values of the known surrounding sample points $X=\left(x_{1} \ldots x_{n}\right)$ with $x_{i} \in \mathbb{R}^{m}$. The corresponding responses $Y=\left(y_{1} \ldots y_{n}\right)$ with $y_{i} \in \mathbb{R}^{p}$ of these experimental points $x_{i}$ are obtained from the complex numerical model simulations. Then, a predicted value $\hat{Y}$ is expressed in two parts as

$\hat{Y}(x)=f(x)+\delta(x)$

where $f(x)$ is the regression function (or trend) and is based on the data, and the Gaussian process $\delta(x)$ is constructed through the residuals with mean 0 , variance $\sigma^{2}$ and a covariance

$\operatorname{Cov}\left(x_{i}, x_{j}\right)=\sigma_{l}^{2} R\left(\theta, x_{i}, x_{j}\right), \quad l=1, \ldots, p$

where $R\left(x_{i}, x_{j}\right)$ is the correlation function, $\sigma_{l}$ is the process variance for the $l$ th component and $\theta$ represents the hyperparameters. The regression function is defined as a multivariate polynomial which takes the form

$f(x)=\sum_{i=1}^{n} \beta_{i} \cdot b_{i}(x)$,

where $\beta_{i}$ denotes the coefficients and $b_{i}(x)$ denotes the basis functions such as the power base for a polynomial. In some Kriging variants, the regression function might be taken as 0 (e.g. simple Kriging) or set to a constant $f(x)=b_{0}$ (e.g. ordinary Kriging). In simple words, the regression function captures the general trend of the data and the Gaussian Process interpolates the residuals. Selecting the correct regression function is a difficult problem; hence, the regression function is often chosen as a constant, which has been proven to be enough for common engineering problems.

The stochastic part shown in Equation (12) includes the correlation function, which is a function of the distance between surrounding data points and the predictor point, respectively. The correlation functions are semivariograms that define the degree of spatial correlation between data points in stochastic processes, a correlation function of 
the type $R\left(\boldsymbol{x}_{\boldsymbol{i}}, \boldsymbol{x}_{\boldsymbol{j}}\right)=R\left(\boldsymbol{x}_{\boldsymbol{i}}-\boldsymbol{x}_{\boldsymbol{j}}\right)$, is generally selected [47], which is largely dependent on the separation distances. The correlation function $R\left(\boldsymbol{x}_{\boldsymbol{i}}, \boldsymbol{x}_{\boldsymbol{j}}\right)$ is to be chosen and several correlation functions are suggested in the literature. The Gaussian and exponential correlation functions are probably the most used, however, others such as the simplified Matérn functions with shape parameter $v=3 / 2$ and $v=5 / 2$, known as Matérn-3/2 and Matérn-5/2 respectively, are also suggested in the literature. Others less common could be the linear, spherical or circular correlation functions.

\subsection{Probabilistic models}

There are many uncertainties related to the assessment of loads and load effects of such complex systems. The dynamic response of an OWT support structure is computed using coupled simulations, which include a large set of parameters that together, ultimately, define the actions on the system.

Here, for the coupled analysis, the uncertainties related to load calculations are accounted through the stochastic variables. Uncertainties related to characterisation of the wind, waves and currents, from measurements to extrapolation methods, are represented through $\chi_{s t}$. The factor $\chi_{R}$ is used in the buckling failure to represent the variability of the resistance model from Equation 11 . The uncertainty related to the Kriging model used to obtain the load effect is incorporated through $\chi_{k} \cdot \chi_{\text {aer }}$ is related to the uncertainty in the assessment of the aerodynamic coefficients. $\chi_{d y n}$ and $\chi_{f}$ represent the uncertainty in modelling the dynamic response and the foundation model respectively. $\chi_{\sigma}$ is used in the Von Mises failure mode and accounts for the uncertainty in the computation of the stresses. $\chi_{\text {sim }}$ introduces the uncertainty related to the limited number of coupled simulations. Thickness at tower base level, yield strength and Young's Modulus are considered stochastic variables as well with their probabilistic models. All these stochastic variables are presented in Table 7-1, and are based on $[21,41]$.

Table 7-1. Probabilistic models of the stochastic models

\begin{tabular}{|c|c|c|c|}
\hline Description & $\begin{array}{l}\text { Distribution } \\
\text { type }\end{array}$ & Mean & $\mathrm{CoV}$ \\
\hline \multicolumn{4}{|l|}{ Random variables } \\
\hline$f_{y} \quad$ Yield stress & Lognormal & $2.35 \mathrm{e} 8 \mathrm{~Pa}$ & 0.05 \\
\hline Young's Modulus & Lognormal & $210 \mathrm{e} 9 \mathrm{~Pa}$ & 0.03 \\
\hline $\begin{array}{ll}\text { Wub } & \begin{array}{l}\text { Wind speed at hub } \\
\text { height }\end{array}\end{array}$ & Normal & $12 \mathrm{~m} / \mathrm{s}$ & 0.05 \\
\hline $\begin{array}{lll}t & \begin{array}{l}\text { Thickness } \\
\text { level }\end{array}\end{array}$ & Normal & $0.04 \mathrm{~m}$ & 0.03 \\
\hline \multicolumn{4}{|l|}{ Other uncertainties } \\
\hline $\begin{array}{ll}\chi_{R} & \text { Resistance model }\end{array}$ & Lognormal & 1 & 0.05 \\
\hline
\end{tabular}




\begin{tabular}{cllll}
$\chi_{k}$ & Kriging model & Lognormal & 1 & 0.1 \\
$\chi_{s t}$ & Limited wind data & Lognormal & 1 & 0.1 \\
$\chi_{\text {aer }}$ & Aerodynamic coefficients & Gumbel & 1 & 0.1 \\
$\chi_{\text {dyn }}$ & Dynamic response & Lognormal & 1 & 0.05 \\
$\chi_{f}$ & Foundation model & Lognormal & 1.1 & 0.05 \\
$\chi_{\sigma}$ & Stress computation & Lognormal & 1 & 0.03 \\
$\chi_{\text {sim }}$ & Limited simulations & Normal & 1 & 0.05 \\
\hline
\end{tabular}

Then, considering the stochastic variable uncertainties from Table 6-1, the limit state equations corresponding to Von Mises, buckling and tower clearance failure modes are written as follows, where the subindex krig indicates a Kriging model. These expressions represent the kriging surface that is fit to the sample points. These are complex and extremely long functions which are not presentable practically in general, hence are treated implicitly.

$$
\begin{aligned}
& g_{V M}=f_{y}-\chi_{k} \chi_{s t} \chi_{a e r} \chi_{d y n} \chi_{f} \chi_{\sigma} \chi_{s i m} \sigma_{V M, k r i g}(V, t) \\
& g_{B k}=\frac{1}{6}\left(1-0.84 \frac{D}{t} \frac{f_{y}}{E}\right)\left(D^{3}-(D-2 t)^{3}\right) f_{y} \chi_{R}-\chi_{k} \chi_{s t} \chi_{a e r} \chi_{d y n} \chi_{f} \chi_{s i m} S_{M, k r i g}(V, t) \\
& g_{C l}=\text { radi }-\chi_{k} \chi_{s t} \chi_{a e r} \chi_{d y n} \chi_{f} \chi_{s i m} t w r_{g a p, k r i g}(V, t)
\end{aligned}
$$

In the limit state function related to the blade-tower clearance (Equation (17)), the load effect, i.e. the Kriging model, is obtained directly from the coupled simulations and indicates the distance between the blade tip and the tower axis. If the distance is zero or lower, it means that the blade is crashing against the tower.

\subsection{Implicit reliability levels}

The implicit reliability levels of a design standard can be reverse engineered by performing a reliability assessment on a structure that has been designed following a given standard and safety factors.

The design load cases 1.3 and 1.6a from the IEC 61400-3 [5] are selected to derive the reliability index of the Upwind structure for the failure modes considered. These two DLCs are chosen based on the list with the most severe load cases from [10]. Note that the three failure modes are treated independently, and no system effects are considered - this corresponds to the basic approach in structural codes based on checking failure modes one at the time.

The DLC 1.3 represents a power production case under a 50-year return period extreme wind turbulence (ETM) and a normal sea state. On the other hand, the DLC 1.6a, while considering normal wind conditions, includes the Severe Sea State (SSS) with a significant wave height as function of the wind speed $H_{S}=H_{S, S S S}(V)$, such that the 
combination of the significant wave height and the wind speed has a recurrence period of 50 years. If data to obtain the SSS is not available, the unconditional extreme significant wave height $H_{S 50}$ with a recurrence period of 50 years can be used as a conservative value for $H_{S}$ independently of the wind speed [4]. Both load cases represent events with a return period of at least 50 years, and that is the reference span of the probabilities of failure derived from these simulations. The reliability indices are commonly compared for a 1-year reference, e.g. implicit annual target reliability in IEC 61400-1 is 3.09, thus the probabilities of failure obtained from the reliability analysis are here transformed from a 50 to a 1-year probabilities of failure as follows:

$P_{f, 1}=1-\left(1-P_{f, 50}\right)^{1 / 50}$

where $P_{f, 1}$ and $P_{f, 50}$ represent the annual probability of failure and the probability of failure for 50 years respectively.

The results of the reliability analysis are shown in Table 7-2. Overall, the numbers show that the structure is over-designed for the three limit state functions and for both the DLCs. The results related to the blade-tower clearance are not shown as the probability of failure was so small that reliability methods did not converge - implying that with these characteristic values the blade tip is very far from the tower. As expected, the LSF related to Von Mises' stress reaching yield has higher probabilities of failure as it is very conservative, the results for the other LSF, however, also yield large $\beta$ s. This may occur for two reasons: the first one being that the parameters defining the geometry might be driven by fatigue cases instead of ULS. The second one is that, maybe, the reliability levels achieved with the current load partial safety factor of 1.35 lead to an overdesigned structure.

The loads at tower base level are expected to be driven by DLC 1.3 because, at this level, loads from the wind are significantly more relevant than the hydrodynamic loads and the DLC 1.3 uses extreme turbulence whereas DLC 1.6 uses normal wind conditions. This is expected to change when the LSFs are studied at seabed level, where the SSS in DLC 1.6a increases the loads from waves. However, after performing the same computations at sea-bed level the results showed negligible probabilities of failure, this is because, although the loads at seabed level increase significantly, so does the tower thickness and with it the yield strength. In this case, the thickness of $8 \mathrm{~cm}$ and the $245 \mathrm{e} 8 \mathrm{MPa}$ of yield strength largely outweigh the load increase.

Table 7-2. Probabilities of failure for different DLCS and LSFs with a reference period of 1 year.

\begin{tabular}{|l|l|l|l|l|}
\hline \multirow{2}{*}{ LSF } & \multicolumn{3}{|c|}{ Reliability index $(\beta)$} \\
\cline { 2 - 5 } & 1 year & 25 years & 1 year & 25 years \\
\hline \multirow{2}{*}{$g_{V M}$} & $\beta=3.55$ & $\beta=3.36$ & $\beta=3.88$ & $\beta=3.71$ \\
& $P_{f}=1.922 \mathrm{e}-4$ & $P_{f}=3.844 \mathrm{e}-4$ & $P_{f}=5.246 \mathrm{e}-5$ & $P_{f}=1.049 \mathrm{e}-4$ \\
\hline
\end{tabular}




\begin{tabular}{|l|l|l|l|l|}
\hline \multirow{2}{*}{$g_{B k}$} & $\beta=5.15$ & $\beta=5.02$ & $\beta=4.9352$ & $\beta=4.79$ \\
& $P_{f}=1.307 \mathrm{e}-7$ & $P_{f}=2.614 \mathrm{e}-7$ & $P_{f}=4.003 \mathrm{e}-7$ & $P_{f}=8.007 \mathrm{e}-7$ \\
\hline
\end{tabular}

Table 7-3 shows the $\alpha$ vectors, which show the sensitivity of the FORM results to the stochastic variables. Note that these are the direction cosines, their absolute value ranges between 0 and 1 and the closer this is to the upper limit, the more significant the influence of the respective random variable is to reliability. A positive value indicates that if the mean and/or standard deviation of the variable is increased, the probability of failure will increase as well, it will be the opposite for the negative sign. As expected, the tower base thickness appears to have the highest impact on general reliability, and overall, the variables defined in the stochastic model with the largest coefficients of variation are the ones with larger sensitivities.

Table 7-3. Sensitivity of the LSF on the stochastic variables.

\begin{tabular}{|c|r|r|r|r|}
\hline \multirow{2}{*}{$\begin{array}{c}\text { Stochastic } \\
\text { variable }\end{array}$} & \multicolumn{2}{|c|}{$g_{V M}$} & \multicolumn{2}{c|}{$g_{B k}$} \\
\cline { 2 - 5 } & DLC 1.3 & DLC 1.6 & DLC 1.3 & DLC 1.6 \\
\hline$f_{y}$ & -0.108 & -0.040 & -0.138 & -0.128 \\
\hline$D$ & - & - & -0.178 & -0.165 \\
\hline$E$ & - & - & -0.019 & -0.018 \\
\hline$\chi_{R}$ & - & - & -0.094 & -0.087 \\
\hline$\chi_{k}$ & 0.203 & 0.077 & 0.152 & 0.141 \\
\hline$\chi_{s t}$ & 0.212 & 0.079 & 0.313 & 0.290 \\
\hline$\chi_{a e r}$ & 0.223 & 0.076 & 0.477 & 0.407 \\
\hline$\chi_{d y n}$ & 0.106 & 0.040 & 0.157 & 0.145 \\
\hline$\chi_{f}$ & 0.193 & 0.072 & 0.285 & 0.264 \\
\hline$\chi_{\sigma}$ & 0.064 & 0.024 & & \\
\hline$\chi_{\text {sim }}$ & 0.105 & 0.039 & 0.152 & 0.141 \\
\hline$V_{\text {hub }}$ & -0.841 & 0.153 & 0.135 & 0.109 \\
\hline$t$ & -0.285 & 0.974 & 0.667 & 0.746 \\
\hline
\end{tabular}

\subsection{Calibration of safety factors}

Here, the methodology for code calibration explained in section 4.2 is applied using the Upwind structure, the probabilistic models from Table 7-1 and the Kriging models obtained in the previous section. For demonstration purposes, the calibration is carried out for the load safety factor while the resistance safety factor $\left(\gamma_{R}\right)$ is assumed to be 1 .

For each failure mode and a prescribed reliability level, the optimisation starts with a given load partial safety factor $\gamma_{L}$. Then, a design-to-limit is performed with the deterministic values of the stochastic variables to obtain the optimal design variable -in this case, the thickness at tower base level. With the resulting value, the corresponding load effect, which is a function of the design variable, is calculated and used in the LSF with the rest of the stochastic variables to which FORM is applied. The optimization is 
run then until the reliability level obtained from this algorithm minimizes the penalty function.

In this case, the calibration is done for each failure mode independently. Note that while using the same LSF, the design load case does not affect the results. Although the optimal value of the design variable may change from one DLC to another in the designto-limit, the reliability outcome will not: by definition, the optimal design variable makes the LSF zero, thus only the probabilistic models will affect the reliability computations.

Results from the code calibration are shown in Table 7-4 for the failure modes related to Von Mises' stress and local buckling and for different 1-year reference period reliability levels. Note that results are shown for both penalty functions from Equation (1) and (2), i.e., PF1 and PF2 respectively. As expected, the safety factors derived for the LSF $g_{V M}$ are larger than for $g_{B k}$. However, while the LSF $g_{V M}$ is a good indicator to compare results, it is very conservative and would not be used for code calibration in the current standards. The local buckling failure would then be driving the design of such structures. In this case, if it is assumed that IEC 61400-3 delivers the same implicit reliability level $\beta_{1}=3.09$ as in IEC $61400-1$, the result of $\gamma_{L}=1.31$ shows a small margin for optimisation. Note that as mentioned in [37], there is no significant variation between the two penalty functions.

Table 7-4. Calibration of the load partial safety factor for different reliability levels.

\begin{tabular}{|c|l|l|l|l|l|l|}
\hline \multirow{2}{*}{ LSF } & \multirow{2}{*}{$\begin{array}{l}\text { Penalty } \\
\text { function }\end{array}$} & \multicolumn{5}{|c|}{$\gamma_{L}$ as function of Target of Reliability index $(\beta)$} \\
\cline { 2 - 7 } & $\beta_{1}=3$ & $\beta_{1}=3.09$ & $\beta_{1}=3.5$ & $\beta_{1}=4$ & $\beta_{1}=4.5$ \\
\hline \multirow{2}{*}{$g_{V M}$} & PF1 & 1.48 & 1.52 & 1.75 & 2.05 & 2.38 \\
\cline { 2 - 7 } & PF2 & 1.48 & 1.53 & 1.75 & 2.05 & 2.39 \\
\hline \multirow{2}{*}{$g_{B k}$} & PF1 & 1.28 & 1.31 & 1.48 & 1.69 & 1.92 \\
\cline { 2 - 7 } & PF2 & 1.29 & 1.32 & 1.48 & 1.69 & 1.92 \\
\hline
\end{tabular}

It is important to highlight that the number of uncertainties and the characteristics of the probabilistic models are the factors that really impact the code calibration. In other words, in the optimization process of the safety factors, maximum effort should be put in reducing the number of uncertainties and in a better understanding of the quantification of their variability, rather than developing optimised reliability methods. Note that efficient reliability methods are, of course, needed as a tool for code calibration, but they will have a rather small effect on the outcome.

In this direction, the results of the code calibration are studied for different levels of uncertainty. First, the same procedure is applied reducing the uncertainties related to resistance models: the CoV of $f_{y}$ and $E$ are reduced to $2 \%$ and $D$ and $t$ to $1 \%$. Then, on top of that, the CoV of the uncertainties $\chi_{s t}, \chi_{a e r}$ and $\chi_{d y n}$ are reduced by half. And finally, the LSF is simplified with one model uncertainty for both the resistance and load effect components. The evolution of the safety factors for these combinations are shown in Figure 7-1. As expected, for a given a reliability level, the three combinations 
lead to a reduction of the safety factor and the differences become large with higher reliability levels. Reducing uncertainties in both load and resistance models give the best results by leaving significant opportunity for safety factor optimisation. Besides, note that while reducing the number of stochastic variables may simplify some analyses, it may also significantly impact the results.

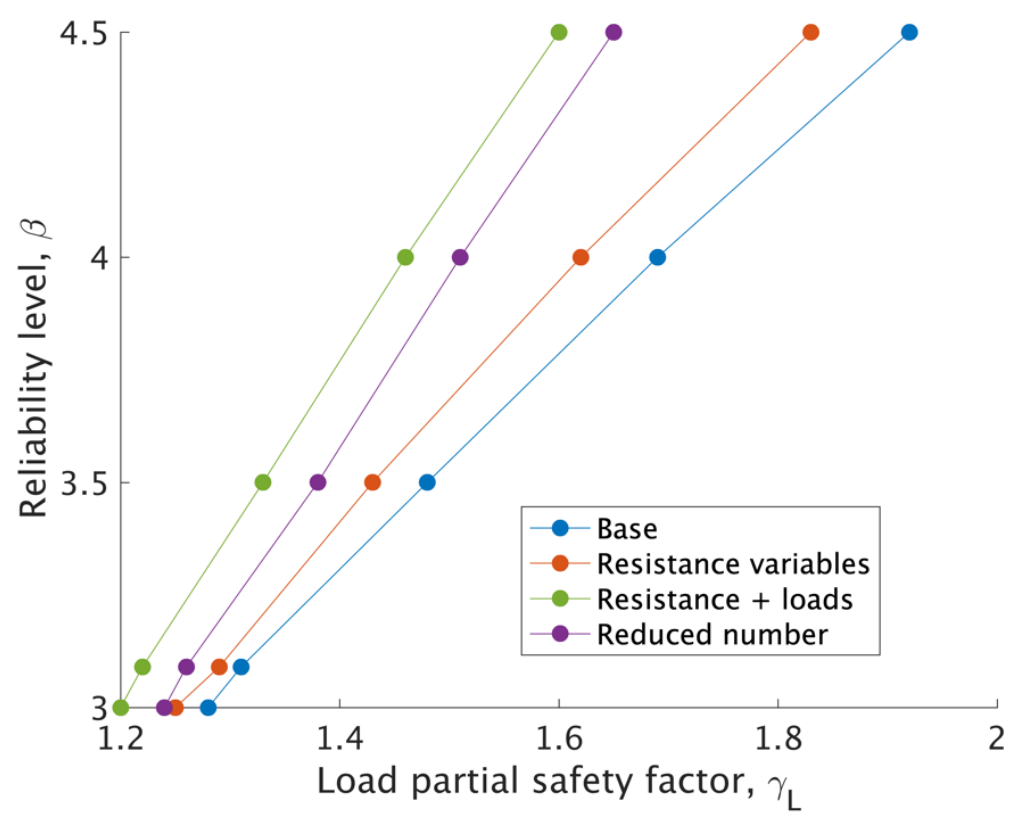

Figure 7-1. Evolution of the safety factors with different levels of uncertainty.

\section{Conclusions}

This paper reviews the state of the art of the safety factors in structural design and specifically on the design of OWTs. Most of the work on calibration of safety factors related to the offshore wind industry has been performed on blades and there is no publicly available research that assessed the validity of the safety factors proposed by the current standards focusing on support structures.

With this in mind, this work reviews the current approaches for reliability-based code calibration of partial safety factors. The chosen method is applied to the $5 \mathrm{MW}$ NREL reference wind turbine mounted atop of the support structure designed in the Upwind project. This structure is chosen because it was designed-to-limit according to the IEC 61400-3, and thus the reliability levels achieved by this code could be derived. Two of the most severe load cases are considered from [10], along with three failure modes: Von Mises' stress reaching yield at tower base level, local buckling at tower base level and blade tip crashing against the tower.

The reliability-based code calibration is demonstrated for each failure mode independently. The results showed that a safety factor of 1.31 was needed for a target reliability of 3.09, which leaves small room for optimisation if the implicit reliability level 
of the IEC 61400-3 is indeed 3.09. Thus, the existing safety factor of 1.35 for ULS in the IEC 61400-3 seems to be adequate. It was also observed that more effort on uncertainty quantification could help to rationalise the safety factors significantly. A different way of approaching the problem could be to tackle the reliability levels currently established by the industry. The combination of the levelized cost of energy decreasing sharply and the fact that OWTs are unmanned structures could lead to a reconsideration of the cost-benefit optimisation of the reliability targets, leading to a potential reduction of the safety factors and thus to more efficient, optimised and cheaper structures.

It is clear that a proper calibration of a standard accounting for all the design load cases, failure modes and system components is highly complex, and is beyond the scope of this research. An example of this complexity is found in the weight associated to a ULS DLC and failure mode in the optimisation function: it should be selected considering how probable failure is under this combination of load case and the failure mode with respect to the other combination of cases and failure modes. This approach requires statistical data regarding failure of the OWT support structures and this information, if it exists at all, is not available in the public domain. Besides, the concept of a DLC is to represent a real situation that the structure may encounter during its design life, even though the probabilities of such an event are small, i.e. cases 1.3 or 1.6 deal with events that have an associated return period of 50-years (ETM, SSS). However, there are deterministic cases such as 1.4 and 1.5 that represent special transients that, not only do not have any associated return period but also, by definition of the probability of a discrete event, the probability of occurrence is 0 . In fact, the authors' could not find any reference that shows the frequency of such events or their existence. The next stages of this research will also focus on safety factor calibration for other types of OWT support structures.

\section{References}

[1] Higgins P, Foley A. The evolution of offshore wind power in the United Kingdom. Renew Sustain Energy Rev 2014;37:599-612. https://doi.org/10.1016/J.RSER.2014.05.058.

[2] Henderson AR, Morgan C, Smith B, Sørensen HC, Barthelmie RJ, Boesmans B. Offshore Wind Energy in Europe- A Review of the State-of-the-Art. Wind Energy 2003;6:35-52. https://doi.org/10.1002/we.82.

[3] Wind Europe. Offshore Wind in Europe: key trends and statistics 2018. 2018.

[4] IEC. IEC 61400-1:2005 Wind turbines - Part 1: Design requirements. Wind Turbines - Part 1 Des Requir 2005;2005:1-92. https://doi.org/10.1055/s-2007-985912.

[5] IEC. IEC 61400-3:2009 Wind turbines: Part 3: Design requirements for offshore wind turbines. 2009.

[6] Jha A, Dolan D, Gur T, Soyoz S, Alpdogan C. Comparison of API \&amp; IEC Standards for Offshore Wind Turbine Applications in the U.S. Atlantic Ocean: Phase II; March 9, 2009 - September 9, 2009. Golden, CO (United States): National Renewable Energy Laboratory (U.S.); 2013. https://doi.org/10.2172/1067926. 
[7] DNV. DNV-OS-J101-Design of offshore wind turbine structures 2004.

[8] API RP 2A-LRFD. Recommended Practice for Planning, Designing, and Constructing Fixed Offshore Platforms-Load and Resistance Factor Design. Am Pet Institute, Washington, DC 2003.

[9] Morató A, Sriramula S, Krishnan N. Kriging models for aero-elastic simulations and reliability analysis of offshore wind turbine support structures. Ships Offshore Struct 2019;14:545-58. https://doi.org/10.1080/17445302.2018.1522738.

[10] Morató A, Sriramula S, Krishnan N, Nichols J. Ultimate loads and response analysis of a monopile supported offshore wind turbine using fully coupled simulation. Renew Energy 2017;101:126-43. https://doi.org/10.1016/J.RENENE.2016.08.056.

[11] Fischer T, De Vries WE, Schmidt B. Upwind design basis (WP4: Offshore foundations and support structures) 2010.

[12] Norton, E; Quarton D. Recommendations for design of offshore wind tubines (RECOFF). D3 Deliv Sensit Stud Doc No 27622003.

[13] API RP 2A-WSD. Recommended Practice for Planning, Designing, and Constructing Fixed Offshore Platforms-Working Stress Design. Am Pet Institute, Washington, DC 2005.

[14] Friis-Hansen P, Sørensen JD. Reliability-based code calibration of partial safety factors. Jt. Comm. Struct. Safety, JCCS-workshop Code Calibration, Zurich: University of Zurich; 2002.

[15] Jiang Z, Hu W, Dong W, Gao Z, Ren Z, Jiang Z, et al. Structural Reliability Analysis of Wind Turbines: A Review. Energies 2017;10:2099. https://doi.org/10.3390/en10122099.

[16] DNV-GL. DNVGL-RP-C203. Fatigue Design of Offshore Steel Structures. Recomm Pract 2016:176.

[17] DNV-GL. DNVGL-RP-C210. Probabilistic methods for planning of inspection for fatigue cracks in offshore structures 2015.

[18] Tarp-Johansen, Niels Jacob and Madsen, Peter Hauge and Frandsen S. Partial safety factors for extreme load effects. 2002.

[19] Tarp-Johansen NJ. Examples of fatigue lifetime and reliability evaluation of larger wind turbine components. 2003.

[20] Toft HS, Branner K, Sørensen JD, Berring P. Reliability-based Calibration of Partial Safety Factors for Wind Turbine Blades 2011:124-8.

[21] Toft HS, Sørensen JD. Reliability-based design of wind turbine blades. Struct Saf 2011;33:333-42. https://doi.org/10.1016/J.STRUSAFE.2011.05.003.

[22] Toft HS, Sørensen JD. Safety Factors - IEC 61400-1 ed. 4 - background document. 2014.

[23] Moriarty P. Safety-factor calibration for wind turbine extreme loads. Wind Energy 2008;11:60112. https://doi.org/10.1002/we.306.

[24] Sørensen JD, Toft HS, Sørensen JD, Toft HS. Probabilistic Design of Wind Turbines. Energies 2010;3:241-57. https://doi.org/10.3390/en3020241.

[25] Agarwal P, Manuel L. Implied Reliability Levels in Different Load Models for Offshore Wind Turbines. Vol. 9B Ocean Renew. Energy, ASME; 2014, p. V09BT09A051. https://doi.org/10.1115/OMAE2014-24598.

[26] Agarwal P, Manuel L. The Influence of the Joint Wind-Wave Environment on Offshore Wind Turbine Support Structure Loads. J Sol Energy Eng 2008;130:031010. https://doi.org/10.1115/1.2931500.

[27] Galambos T V., Ravindra MK. The basis for load and resistance factor design criteria of steel building structures. Can J Civ Eng 1977;4:178-89. https://doi.org/10.1139/177-023.

[28] Melchers RE (Robert E., Beck AT. Structural reliability analysis and prediction. Third Edit. John Wiley \\& Sons; 2018.

[29] Sørensen JD, Kroon IB, Faber MH. Optimal reliability-based code calibration. Struct Saf 
1994;15:197-208. https://doi.org/10.1016/0167-4730(94)90040-X.

[30] Thoft-Cristensen, P and Baker MJ. Structural reliability theory and its applications. Springer Science \\& Business Media; 2012.

[31] Diamantidis D. Report 32: Probabilistic Assessment of Existing Structures-A publication for the Joint Committee on Structural Safety (JCSS). vol. 32. RILEM publications; 2001.

[32] Matthies HG, Brenner CE, Bucher CG, Guedes Soares C. Uncertainties in probabilistic numerical analysis of structures and solids-Stochastic finite elements. Struct Saf 1997;19:283-336. https://doi.org/10.1016/S0167-4730(97)00013-1.

[33] Ditlevsen O. Structural reliability codes for probabilistic design - a debate paper based on elementary reliability and decision analysis concepts. Struct Saf 1997;19:253-70. https://doi.org/10.1016/S0167-4730(97)00009-X.

[34] Vrouwenvelder T. The JCSS probabilistic model code. Struct Saf 1997;19:245-51.

[35] Faber MH, Sørensen JD. Reliability-Based Code Calibration: The JCSS Approach. 9th Int. Conf. Appl. Stat. Probab. Civ. Eng., Millpress; 2003, p. 927-35.

[36] Madsen, Henrik O and Krenk, Steen and Lind NC. Methods of structural safety. Courier Corporation; 2006.

[37] Ditlevsen O, Madsen HO. Structural Reliability Methods. Wiley New York; 1996.

[38] Sørensen JD. Optimal, reliability-based code calibration. AMAS Course Reliab Optim 2002:35-150.

[39] Rackwitz R. A new approach for setting target reliabilities. IABSE. Safety, Risk Reliab. - Trends Eng. Malta 2001, Zurich: IABSE; 2001, p. 531-6.

[40] Nathwani, J. S.; Lind, N. C.; Pandey MD. Affordable Safety by Choice: The Life Quality Methods 1997.

[41] JCSS. Probabilistic model code 2001.

[42] De Vries WE. Upwind Final report WP 4.2: support structure concepts for deep water sites: deliverable D4. 2.8 (WP4: offshore foundations and support structures). 2011.

[43] Yang H, Zhu Y, Lu Q, Zhang J. Dynamic reliability based design optimization of the tripod substructure of offshore wind turbines. Renew Energy 2015;78:16-25. https://doi.org/10.1016/J.RENENE.2014.12.061.

[44] Sørensen JD, Tarp-Johansen NJ. Reliability-based Optimization And Optimal Reliability Level of Offshore Wind Turbines. Int J Offshore Polar Eng 2005;15.

[45] DNV. DNV-RP-C202 Buckling Strength of Shells 2013.

[46] AISC. Specification for structural steel buildings 2005:IL. ANSI/ASIC 360-05.

[47] Sacks J, Welch WJ, Mitchell TJ, Wynn HP. Design and Analysis of Computer Experiments. Stat Sci n.d.;4:409-23. https://doi.org/10.2307/2245858. 